\title{
What's So Social about the Social Brain?
}

\section{R.I.M. Dunbar}

Department of Experimental Psychology

University of Oxford

South Parks Rd

Oxford OX1 3UD

UK

[email: robin.dunbar@psy.ox.ac.uk] 


\section{Summary}

In trying to understand the neurobiology of sociality, we face a problem over the kinds of behavioural indices we can use in experiments. The focus of most research has been on economic games or the socio-cognitive processes involved in dyadic interactions (e.g. theory of mind or prosocial/altruistic behaviour). These largely reflect causal interactions between strangers. However, the nature of primate (and hence human) sociality, as reflected in the social brain hypothesis, is more complex and involves long term bonded relationships that allow individuals to maintain several social layers in their network simultaneously. Identifying suitable tests that can be used in either cognitive or neuroimaging experiments is challenging. 


\section{Introduction}

The last decade has witnessed the dramatic growth of social (sometimes social cognitive) neuroscience. There has been considerable interest, for example, in microeconomic games (mainly various forms of public good games, such as the Prisoner's Dilemma, the Ultimatum Game and Trust Game, among others) in which individuals decide whether or not to act generously towards another individual. On the cognitive side, there has been a longstanding interest focussed on those aspects of cognition thought to underpin autistic spectrum disorders: this has led to the development of a consensus view that there is a mentalising (or theory of mind) network comprising units in the prefrontal cortex, the temporal cortex and the temporo-parietal junction.

The bulk of this research has, however, focussed mainly on dyadic interactions or on cognitive processes thought to be important in dyadic interactions. Economic games are designed mainly to be played between two players, and most of the work on mentalising has focussed on conventional theory of mind (second order intentionality) which asks whether one individual can understand another's mental state. This has been a perfectly sensible research strategy, not least because some aspects of our social behaviour do involve dyadic interactions of this kind. However, both approaches have significant limitations in that, in effect, they focus on the bricks and mortar of sociality, and do not address the real complexity of sociality in primates. Their view of sociality is really the kind of dyad-based sociality that we find in many antelope and deer - species that form socially amorphous herds based on rather transient interactions between pairs of individuals. These kinds of social groups contrast radically with the structured groups that we find in primates, where triadic and polyadic relationships, and to some extent the group itself, become important.

The classic approaches are perfectly reasonable in the context of the research on which they are based. Economic games are premised on the assumption that all human 
relationships are essentially trading relationships: the secondhand car salesman's view of sociality. This encourages us to be concerned about being cheated, or whether the person with whom we strike a deal is likely to renege on the agreement. Indeed, in many experimental contexts, the odds are deliberately stacked to make freeriding an attractive strategy. In most cases, relationships are assumed to be of very short duration: I offer you a commodity or an investment bid, which you can accept or decline in the light of your broad assessment of the risks of being cheated, and after that we seldom meet again. Typically, these games focus on interactions between strangers and almost never concern themselves with other kinds of relationships (friendship, kinship) or with the central aims of sociality (namely, creating functional communities whose purpose is to enable us to solve the larger scale problems that threaten to undermine our ability to survive and reproduce successfully).

Similarly, the principal focus of theory of mind studies has been with the competences of 5-6 year old children. For reasons that remain slightly puzzling, theory of mind (i.e. second order intentionality, as defined by the ability to solve standard false belief tasks) seems to have been taken as the quintessential social cognitive ability, even though adults are capable of handling much higher order intentionality tasks (Kinderman et al. 1998; Stiller \& Dunbar 2007). Theory of mind is, of course, a major rubicon that differentiates humans from all other animals (with the possible exception of great apes: O'Connell \& Dunbar 2003; Cartmill \& Byrne 2007). Theory of mind requires us to be able to imagine what another individual is thinking, and is, of course, critical in our dyadic interactions - not least because it is fundamental to our being able to hold a conversation with another individual (we speak telegraphically and in metaphors, and these require the listener to figure out what it is the speaker means to say). However, a significant proportion of our conversations involve several individuals simultaneously (the average size of conversation groups is around 3 individuals, often with a fourth person as the object of the conversation) (Dunbar et al. 1995; 
Dezecaches \& Dunbar 2013), and many examples of culture (e.g. staged drama, novels) require the audience to handle the mind states of several characters at once (Stiller et al. 2004; Krems \& Dunbar 2013).

I want to argue that, in effect, these conventional approaches to social neuroscience focus on the bricks and mortar of sociality, the raw components that go towards making the primate (and human) social world as complex as it is. What they do not address is the building that these bricks are used to construct and, to pursue the metaphor, the problem is that the bricks alone do not define the building. I will try to suggest what this complexity actually involves in the hopes that it will stimulate new approaches. I will begin, however, by briefly reviewing the social brain hypothesis and the evidence for it, and will then use this as a platform to explore the nature of primate sociality.

\section{The social brain hypothesis}

As a concept, the social brain hypothesis has two distinct origins. The term was originally used in cognitive neuroscience (notably by Brothers XXXX) to capture a sense that much of the brain's activity is really geared to steering a way through the complex social world in which we live. This sense was picked up in the comparative literature when it was explicitly used as an explanation for the unusually large brains of primates compared to all other vertebrates (including other mammals). Originally framed as the Machiavellian Intelligence Hypothesis, it argued that primates need large brains because they live in unusually complex societies (Byrne \& Whiten 1988). Since there were some concerns about the implication that primates engaged in genuinely political strategies, the name was soon changed and Brothers' term adopted instead (Dunbar 1992; Barton \& Dunbar 1997). In some senses, this was an appropriate development because the Machiavellian Hypothesis seemed to imply that there was simply a qualitative difference between primates and all other species, 
and no difference among the primates themselves. In fact, primates have brains that vary considerably, both in terms of absolute size and relative to body size, and this itself requires an explanation. The social brain hypothesis at least provides the basis for differences between species. It also provides a basis for differences between different mammalian families.

The core evidence adduced in support of the social brain hypothesis is the fact that there is a relationship between social group size in primates (including humans) and the volume of the neocortex (Dunbar 1992; Barton 1996), or better still the frontal lobes of the neocortex (Dunbar 2011) (Fig. 1). Subsequent comparative analyses have confirmed and extended this finding in a number of important ways. First, Dunbar \& Shultz (2007) undertook a path analysis that explored the intercorrelations between brain size and a number of anatomical and behavioural variables in primates, and concluded that the main predictor of neocortex volume was social group size, with other ecological and anatomical variables being either constraints on brain growth or functional consequences of large brains. Second, Shultz \& Dunbar (2010a) showed that, across the major mammal families, encephalisation across evolutionary time (i.e. the rate with which brain size has increased relative to body size across geological time) correlates with the frequency of bonded social systems among the living representatives of each family. Third, the latter relationship broadly reflects the fact that, outside primates, bonded social systems in mammals typically involve pairbonded monogamy, such that the main driver of the social brain in non-primate mammals is in fact pairbonding (Shultz \& Dunbar 2007) and the cognitive demands of maintaining long lasting pairbonds (Dunbar \& Shultz 2010).

The equation relating neocortex volume and group size in great apes was originally used to predict group size for humans, and gave a value of $~ 150$ (Dunbar 1993). Evidence to support the claim that this is a 'natural' group size for humans came from quantitative data on both the size of communities in ethnographic societies and the size of personal (i.e. ego- 
centric) social networks. Subsequent analyses of these data revealed that, in fact, human social networks are highly structured, such that the community of 150 in fact consists of a series of layers that are scaled with a regular scaling ratio of about three (Zhou et al. 2005). In other words, both ego-centric networks and social organisations consist of layers or groupings of that contain, inclusively, about 5, 15, 50 and 150 individuals, with this pattern continuing on through at least two further layers (at 500 and 1500).

Since these original data were published, a number of neuroimaging studies have confirmed and extended the relationship between social group size (indexed as personal social network size) and brain size at the level of the individual. In a series of analyses, Powell et al. $(2010,2012)$ demonstrated that, in individual adult humans, social network size (indexed as the 15-layer) correlated with both an individual's mentalising competences and the gross volume of their orbitofrontal cortex, while Lewis et al. (2011) demonstrated that network size and mentalising competences also correlated parametrically with the volume of core frontal and temporal regions in the theory of mind network, with the ventromedial prefrontal cortex providing the focus for a uniquely strong three-way correlation. Subsequently, Kanai et al. (2012) found similar correlations between the number of friends listed on subjects' preferred social networking site and the volume of various frontal and temporal cortex regions. Perhaps more importantly, Sallet et al. (2012) demonstrated a similar set of relationships in macaques (with social network size indexed as housing group). In an earlier analysis of humans, XXXX et al (2011) had used a somewhat idiosyncratic definition of social networks to test for (and confirm) a relationship between the number of friends included in personal networks and the size of the amygdala. Although the amygdala has not featured in either the comparative analyses or other neuroimaging studies, in fact such a relationship is quite plausible: social relationships involve the assessment of emotional cues, and the capacity to interpret these correctly must be important for coping with life in 
large social groups. More importantly, however, the amygdala does have a direct neural connection with the orbitofrontal cortex, and is thus likely to play an ancillary relationship in the extended socialising neural network.

\section{The primate social world}

The social brain data suggest that large brained species (and, indeed, individuals with larger prefrontal cortex elements) can manage more relationships simultaneously than smaller-brained species. This significantly raises the game in terms of the cognitive mechanisms involved. This is not just a matter of remembering who is who (although memory for faces is obviously an important requirement), but rather one of being able to place these individuals in a multidimensional social space such that an animal can assess and manage triadic relationships (how my behaviour towards you will be interpreted by - and affect the behaviour of - a third party), as well as higher order polyadic relationships.

Passingham \& Wise (2013) have argued that anthropoid primates have evolved entirely novel regions of the prefrontal cortex that allow them to undertake very fast one-trial learning, in effect by being able to predict into the future on the basis of very limited information in the past. In contrast, other vertebrates (and this includes prosimian primates) rely on computationally more cumbersome trial-and-error association learning. This prefrontal cortex effect also involves increased capacity to inhibit prepotent responses (Shultz \& Dunbar 2010b). The ability to inhibit prepotent responses is central to living in large social groups both because it enables animals to defer short term gains in the interests of larger long term ones (crucial for group-level solutions to ecological problems) and also because it probably allows animals to hold back on punishment in circumstances where punishing defaulters might have negative consequences for the social stability of a group. Since punishment has been viewed as the archetypal social interaction both by economists and by 
those who have used economic games as an experimental index of sociality, the suggestion that there is a need to withhold punishment in the interests of not destabilising relationships potentially places something of a question mark over this whole approach.

I noted above that bonded relationships seem to be important in driving the evolution of large brains in mammals as a whole. We have some difficulty defining exactly what is involved in these kinds of relationships (Dunbar \& Shultz 2010), in part because we have no real metric for quantifying them, even though most ethologists have no trouble recognising them in practice - and no more so than we do in everyday social life. This may reflect the fact that relationships have a major emotional content rather than being cognitive phenomena, and we have trouble translating experienced emotions into words. The key, however, is that such relationships are long lasting (they may last an entire lifetime), commonly involve a great deal of very intense social grooming (such that the amount of grooming animals do correlates with the size of the group: Lehmann et al. 2007) and often provide the basis for mutual defence against conspecifics (and occasionally even predators).

The bonded relationships that characterise primate sociality are created through what is, in effect, a two step process. Monkeys and apes universally use social grooming as the principal means for expressing social relationships. Although grooming certainly serves to maintain the fur by removing debris and skin flakes (and undoubtedly had it evolutionary origins in hygiene), in reality most of these species groom each other for far longer than is minimally necessary to maintain clean fur. Grooming triggers the release of endorphins (specifically $\beta$-endorphins) (Keverne et al. 1989; Depue \& Morrone-Strupinsky 2005) and these seem to create an internal psychopharmacological platform off which a second (cognitive) mechanism creates the long term, stable relationships of trust and obligation tat provide the basis for coalitions and alliances. Although a great deal has been made of the role of oxytocin in creating social bonds in mammals (including humans), in reality its effects are 
short-lived and lead to rapid habituation (Dunbar 2010), and may only have these effects in a limited range of contexts that apply broadly across the mammals (e.g. limited term pairbonds and transient interactions). It has been explicitly argued that because the oxytocin mechanism is relatively weak, the evolution of bonded relationships in primates required a more robust pharmacological mechanism to support this new form of sociality, and that this mechanism is the endorphin system (Broad et al. 2006; Machin \& Dunbar 2011).

Some evidence that the complexity of relationships varies in a consistent way with neocortex size is provided by the original social brain data - the plot of group size against neocortex volume. These data turn out to consist of several (in fact, four) distinct grades, of which only the ape grade is obvious to the naked eye although the others can be shown statistically (Fig. 1). What this tells us is that the computational demands of sociality increase progressively across the grades, with ape sociality apparently being more cognitively demanding than that of any other simian and prosimian forms of sociality. In a nutshell, apes require a bigger computer to manage a group of a given size than the most social monkeys do, and these in turn require a bigger one that the less social monkeys, etc. We do not know exactly why ape sociality is so much more demanding than monkey sociality, but the fact is that it is. What we do know is that species that have larger neocortices have larger grooming cliques (Kudo \& Dunbar 2001), are more likely to use coalitions in mutual defence (Dunbar \& Shultz 2007), are more likely to use tactical deception (Byrne \& Corp 2004) and are likely to exploit more sophisticated male mating strategies (Pawlowski et al. 1998).

More importantly, the structure of social groups varies systemically with brain size. Using grooming frequencies as an index of social relationships between individuals (a standard metric in primatology), Lehmann \& Dunbar (2009) have shown that larger social groups become increasingly substructured in large-brained species of Old World monkeys (Fig. 2). This represents a rather complex relationship between group size, brain size, 
grooming time and network complexity. In effect, although animals in large groups spend more time engaged in social grooming, this is not because they groom with more individuals. In fact, as group size and grooming time increase, the number of animals groomed actually decreases: the additional grooming time becomes concentrated on fewer individuals, creating stronger bonds as a result since, as we have shown for humans, the time invested in a relationship directly affects it quality (Roberts \& Dunbar 2011a,b). In species with relatively small brains, animals simply try to groom with every new group member, and ultimately this places a limit on the size of group that can be bonded together. In species with relatively big brains, however, individuals don't try to groom with everyone, but instead withdraw from casual interactions to focus more and more of their grooming on their core grooming partners as group size increases. In less intensely social species, this would result in a reduction in group size, but in these very social species it provides a mechanism that allows them to support ever bigger groups.

In effect, these species somehow manage to create a two-tier form of sociality: they can maintain a larger superficial structure (i.e. a larger group) by investing more heavily in an inner core of relationships even though they don't invest directly in the outer layer. In other words, they can manage two different kinds of relationships at the same time - what amount to weak and strong ties in the Granovetter (1973) sense. We know that primates can manage to distinguish, and keep separate, two different dimensions to a relationship (kinship versus rank) (Bergman et al. 2003). Our network analyses suggest that they can also manage two separate kinds of relationships as well.

All this perhaps makes more sense in the light of the function of social groups. In primates, social groups are the principal defence against predators, as they are in many mammals. However, in herd-forming mammals, groups are relatively ephemeral, increasing or decreasing in size as a consequence of the moment-by-moment changes in predation risk 
and the costs of being in the group. These costs relate in part to stresses created by the proximity of other individuals (which have very significant effects on female menstrual endocrinology, resulting in infertility: Abbott et al. 1986; Dunbar 1988) and in part to the fact that living in larger groups forces animals to forage for longer and travel further each day (Dunbar et al. 2009). Such groups lack stability, and lack collective defence: animals do not go to each other's defence. Their value as defensive alliances is limited because the members can easily disperse when the stresses become too great: they have no intrinsic 'loyalty' to each other or to the group as an abstract entity (this last being a particular feature of human sociality).

Among the cercopithecine monkeys, there is, in effect, an intrinsic commitment to the group as a group. Females are philopatric (remain in their social group for life) and this makes it difficult an individual to leave or join simply because the costs of being in the group rise. In effect, it is locked into an implicit social contract which requires it to put up with short term costs (the stresses noted above, and the need to resist taking short term advantage of other group members) in order to benefit from the greater benefits (in terms of protection from predation) provided in the longer run by the group as a whole. This trade off seems to require quite sophisticated cognitive abilities.

\section{The Challenge}

Finding suitable measures that reflect these more complex kinds of sociality is not going to be easy. What is clear, however, is that the kinds of tests that are in wide current use are not satisfactory: they reflect transient interactions between strangers rather than the kinds of long term bonded relationships that give primate (and hence human) sociality is peculiar feel. Part of the problem here is that relationships are emotional and it seems that the emotional component of relationships is something we don't have good cognitive access to 
and hence have trouble describing in language. The fact that we cannot verbalise about or comment on the emotional component of relationships means that we don't have a good metric for comparing relationships of different quality (either between species or, within species, between individuals) and this makes it difficult for us to study them (Dunbar \& Shultz 2010). As a result, perhaps, we tend to focus our attention on low level cognitive processes or behavioural interactions that may not capture the real underlying nature of humans (and primate) sociality. This poses a challenge to which we urgently need to find a solution. 


\section{References}

Abbott DH, Keverne EB, Moore, GF, Yodyinguad U (1986) Social suppression of reproduction in subordinate talapoin monkeys, Miopithecus talapoin. In: Else J, Lee PC (eds) Primate Ontogeny. Cambridge, Cambridge University Press, pp. 329-341

Barton RA (1996) Neocortex size and behavioural ecology in primates. Proceedings of the Royal Society, London, 263B: 173-177

Barton RA, Dunbar RIM (1997) Evolution of the social brain. In: Whiten A, Byrne R (eds) Machiavellian Intelligence II. Cambridge, Cambridge University Press, pp. 240-263

Bergman TJ, Beehner JC, Cheney DL, Seyfarth RM (2003) Hierarchical classification by rank and kinship in baboons. Science 302: 1234-1236

Broad KD, Curley JP, Keverne EB (2006) Mother-infant bonding and the evolution of mammalian social relationships. Philosophical Transactions of the Royal Society, London, 361B: 2199-2214

\section{Brothers, L.}

Byrne RW, Corp N (2004) Neocortex size predicts deception rate in primates. Proceedings of the Royal Society, London, 271B: 1693-1699

Byrne R, Whiten A (1988) The Machiavellian Intelligence Hypothesis. Oxford, Oxford University Press

Cartmill EA, Byrne RW (2007) Orangutans modify their gestural signaling according to their audience's comprehension. Current Biology 17: 1345-1348

Depue RA, Morrone-Strupinsky JV (2005) A neurobehavioral model of affiliative bonding: implications for conceptualizing a human trait of affiliation. Behavioral and Brain Sciences 28: $313-395$

Dezecache G, Dunbar RIM (2013) Sharing the joke: the size of natural laughter groups. Human Nature 
Dunbar RIM (1988) Primate Social Systems. London, Chapman \& Hall

Dunbar RIM (1993) Coevolution of neocortex size, group size and language in humans. Behavioral and Brain Sciences 16: 681-735.

Dunbar RIM (2010) The social role of touch in humans and primates: behavioural function and neurobiological mechanisms. Neuroscience and Biobehavioral Reviews 34: 260-268

Dunbar RIM (2011) Evolutionary basis of the social brain. In: Decety J, Cacioppo J (eds) Oxford Handbook of Social Neuroscience. Oxford, Oxford University Press, pp. 2838

Dunbar RIM, Shultz S (2007) Understanding primate brain evolution. Philosophical Transactions of the Royal Society, London, 362B: 649-658

Dunbar RIM, Shultz S (2010) Bondedness and sociality. Behaviour 147: 775-803

Dunbar RIM, Duncan N, Nettle D (1995) Size and structure of freely forming conversational groups. Human Nature 6: 67-78

Dunbar RIM, Korstjens AH, Lehmann J (2009) Time as an ecological constraint. Biological Reviews 84: 413-429

Granovetter M (1973) The strength of weak ties. American Journal of Sociology 78: 13601380

Kanai R, Bahrami B, Roylance R, Rees G (2011) Online social network size is reflected in human brain structure. Proceedings of the Royal Society, London 279B: 1327-1334

Keverne EB, Martensz ND, Tuite B (1989) Beta-endorphin concentrations in cerebrospinal fluid of monkeys are influenced by grooming relationships. Psychoneuroendocrinology 14: 155-161

Kinderman P, Dunbar RIM, Bentall RP (1998) Theory-of-mind deficits and causal attributions. British Journal of Psychology 89: 191-204 
Krems JA, Dunbar RIM (2013) Clique size and network characteristics in hyperlink cinema: constraints of evolved psychology. Human Nature (in press).

Kudo H, Dunbar RIM (2001) Neocortex size and social network size in primates. Animal Behaviour 62: 711-722

Lehmann J, Dunbar RIM (2009) Network cohesion, group size and neocortex size in femalebonded Old World primates. Proceedings of the Royal Society, London, 276B: 44174422

Lehmann J, Korstjens A, Dunbar RIM (2007) Group size, grooming and social cohesion in primates. Animal Behaviour 74: 1617-1629

Lewis PA, Rezaie R, Browne R, Roberts N, Dunbar RIM (2011) Ventromedial prefrontal volume predicts understanding of others and social network size. NeuroImage 57: $1624-1629$

Machin A, Dunbar RIM (2011) The brain opioid theory of social attachment: a review of the evidence. Behaviour 148: 985-1025

O’Connell S, Dunbar RIM (2003) A test for comprehension of false belief in chimpanzees. Evolution and Cognition 9: 131-139

Passingham RE, Wise SP (2012) The Neurobiology of the Prefrontal Cortex. Oxford, Oxford University Press.

Pawlowski BP, Lowen CB, Dunbar RIM (1998) Neocortex size, social skills and mating success in primates. Behaviour 135: 357-368

Powell J, Lewis PA, Dunbar RIM, García-Fiñana M, Roberts N (2010) Orbital prefrontal cortex volume correlates with social cognitive competence. Neuropsychologia 48: $3554-3562$

Powell J, Lewis PA, Roberts N, García-Fiñana M, Dunbar RIM (2012) Orbital prefrontal cortex volume predicts social network size: an imaging study of individual differences 
in humans. Proceedings of the Royal Society, London, 279B: 2157-2162

Roberts SBG, Dunbar RIM (2011a) The costs of family and friends: an 18-month longitudinal study of relationship maintenance and decay. Evolution and Human Behavior 32: 186-197

Roberts SBG, Dunbar RIM (2011b) Communication in social networks: effects of kinship, network size and emotional closeness. Personal Relationships 18: 439-452

Sallet J, Mars RB, Noonan MP, Andersson JL, O’Reilly JX, Jbabdi S, Croxson SP, Jenkinson M, Miller KL, Rushworth MFS (2011) Social network size affects neural circuits in macaques. Science 334: 697-700

Shultz S, Dunbar RIM (2007) The evolution of the social brain: Anthropoid primates contrast with other vertebrates. Proceedings of the Royal Society, London, 274B: 2429-2436

Shultz S, Dunbar RIM (2010a) Encephalisation is not a universal macroevolutionary phenomenon in mammals but is associated with sociality. Proceedings of the National Academy of Sciences, USA, 107: 21582-21586

Shultz S, Dunbar RIM (2010b) Species differences in executive function correlate with hippocampus volume and neocortex ratio across non-human primates. Journal of Comparative Psychology 124: 252-260

Stiller J, Dunbar RIM (2007) Perspective-taking and memory capacity predict social network size. Social Networks 29: 93-104

Stiller J, Nettle D, Dunbar RIM (2004) The small world of Shakespeare's plays. Human Nature 14: $397-408$

Zhou W-X, Sornette D, Hill RA, Dunbar RIM (2005) Discrete hierarchical organization of social group sizes. Proceedings of the Royal Society, London, 272B: 439-444 


\section{Legends to Figures}

Fig. 1. Mean social group size for a species plotted against its neocortex ratio (volume of neocortex divided by the volume of the rest of the brain), showing four separate socio-cognitive grades. (Redrawn from Dunbar 1992, 2011)

Fig. 2. Network density among females (indexed by dyadic grooming frequencies) as a function of relative neocortex volume for cercopithecine monkey species. Network density indexes the interconnectedness of group members, and can be interpreted as showing increased clumpiness when low. Reproduced with the permission of the publishers, the Royal Society of London, from Lehmann \& Dunbar (2009). 
Dunbar - Figure 1

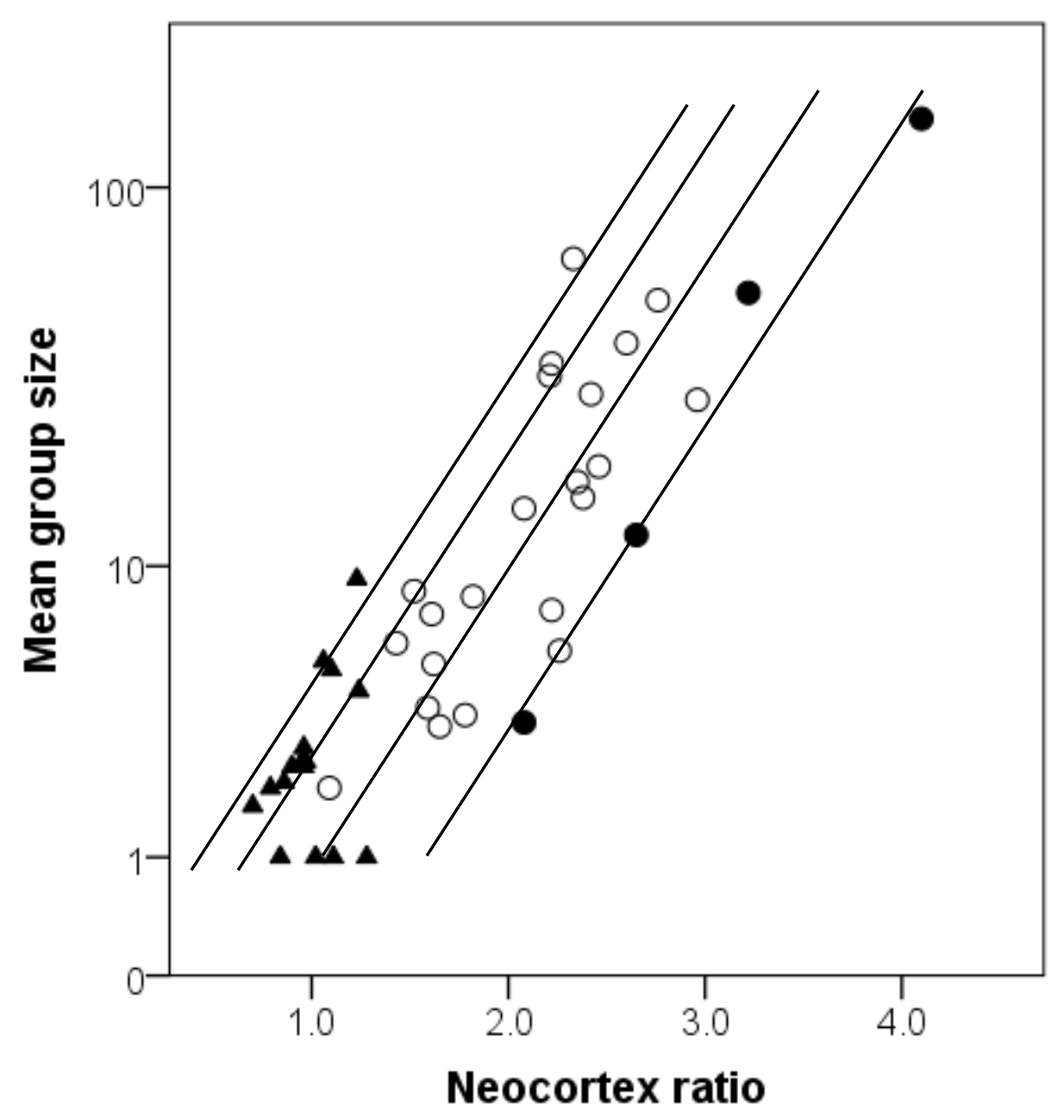


Dunbar - Figure 2

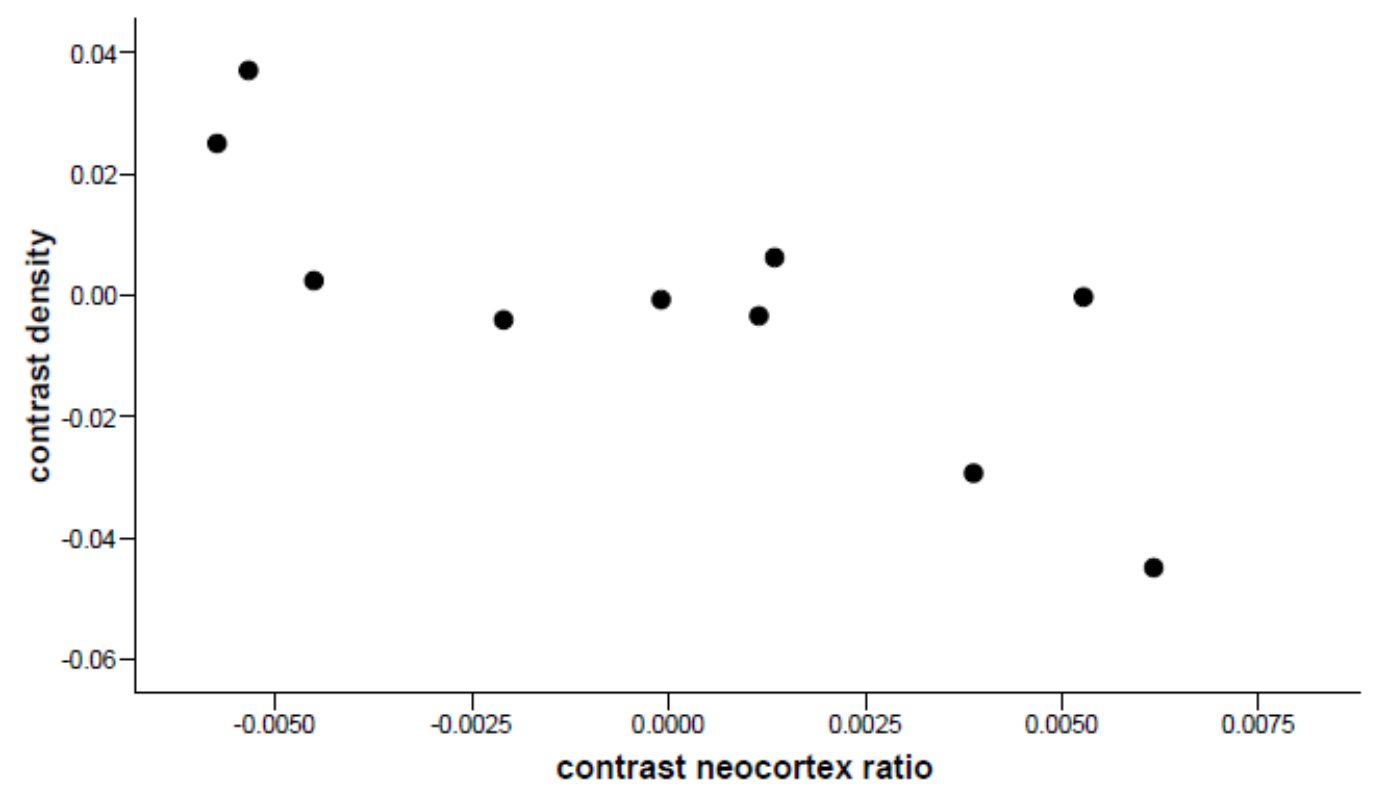

